\title{
(Re)significación de la práctica docente: reflexiones acerca del quehacer del maestro
}

\section{A new meaning of the teaching practice: reflections on the teacher's daily basis}

\author{
Leonor Itsmenia Granados Becerra* \\ Luis Eduardo Martinez Méndez** \\ Sonia Lucía Romero Alfonso ***
}

Fecha de recepción: 12 de Febrero de 2016

Fecha de aprobación: 5 de Septiembre de 2016

Artículo de Investigación

\section{Resumen}

El presente artículo busca reflexionar sobre la práctica docente de los profesores (autores) de matemáticas y ciencias naturales y educación ambiental de la Institución Educativa Juan José Rondón de Soatá para que afronten cuestionamientos y de manera crítica, permanente, constante autoevalúen su quehacer, con el objetivo de (re)significar estas prácticas docentes, al materializar estrategias hibridas para propiciar en el aula otras interacciones, otros sentidos en ellas. La investigación se desarrolla en cuatro fases que interactúan, teniendo en cuenta la espiral de Carr y Kemmis, planificación, acción, observación y reflexión, se desarrolla a partir del paradigma sociocrítico, cuenta con una unidad de análisis de 28 estudiantes de grado 802 .
*Institución Educativa Juan José Rondón de Soatá, Boyacá-Colombia granadosleo@hotmail.com **: Institución Educativa Juan José Rondón de Soatá, Boyacá-Colombia

luiseduardotunja@yahoo.es *** Universidad Pedagógica

y Tecnológica de Colombia, Boyacá-Colombia sonia.romero@uptc.edu.co

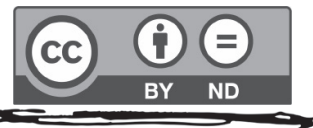


En síntesis, se espera del docente la construcción de conciencia sobre la importancia de (re)significar su labor, pensar, analizar, comprender y, desde esta interpretación, generar cambios que incidan tanto en el mejoramiento de su desempeño como en el bienestar de los estudiantes en términos de aprendizajes. La (re)significación parte de la reflexión y autocrítica, en ella se da sentido a lo que se hace y este sentido es nuevo; es decir, se rehace, repiensa y reelabora cada una de las actividades que se realicen, buscando otras dinámicas respecto del componente didáctico de la práctica docente en beneficio del proceso de aprendizaje de los estudiantes.

Palabras Clave: práctica docente, (re)significación, educación, cultura, sociedad, enseñanza, aprendizaje.

\section{Abstract}

This article seeks to reflect on the teaching practice of the professors (authors) of mathematics and natural sciences of the Juan José Rondón Educational Institution in Soatá (Colombia) to face questions, to critically, permanently and constantly self-assess their work, with the objective of giving a new meaning to these teaching practices, to materialize hybrid strategies to promote other interactions in the classroom. The research is developed in four phases that interact, taking into account the spiral of Carr and Kemmis, planning, action, observation and reflection, is developed from the socio-critical paradigm, has a unit of analysis of 28 students of eighth grade. In short, the teacher is expected to build awareness about the importance of giving a new meaning to their work, think, analyze, understand and, from this interpretation, generate changes that affect both the improvement of their performance and the welfare of the students in terms of learning. The (re) significance part of the reflection and self-criticism, it gives meaning to what is done and this sense is new; that is to say, each of the activities is recreated, re-thought and re-elaborated, looking for other dynamics regarding the didactic component of the teaching practice in benefit of the students' learning process.

Keywords: teaching practice, education, culture, society, teaching, learning. 


\section{Introducción}

Las siguientes líneas pretenden hacer un acercamiento a la práctica docente como proceso dinámico en el que intervienen diversas dimensiones que la enriquecen, la construyen, y a la vez, son el punto de partida para adelantar un análisis provechoso, en relación con la multiplicidad de oportunidades de mejoramiento que tiene el docente frente a sí mismo para mejorarla y reconstruirla diariamente. Se comenzará por abordar su definición según los aportes expuestos por autores como Fierro, Fortoul y Rosas (1999). En seguida, se realiza una aproximación sobre las seis dimensiones que la constituyen (personal, institucional, interpersonal, social, didáctica y valorar), y cómo estas se entrelazan dinámicamente en una relación pedagógica, así como la nombran las autoras.

En consecuencia, observar en el docente un líder pedagógico, habilitado para aportar y en esa misma vía innovar en los procesos dados al interior de las instituciones educativas; asunto que adquiere una importancia visible. Por tal razón, algunas de las ideas expuestas girarán en torno a esta proyección, para conducir la exposición hacia la descripción de experiencias reales que, adelantadas desde un enfoque de investigación cualitativa y siguiendo una línea de investigación-acción, según Car \& Kemmis (1988), han sido de gran significado en el continuo proceso de formación de los autores.

Al finalizar, se dejarán a manera de conclusión, algunas ideas de aquello que no debe perderse de vista cuando se trata de pensar en la práctica docente como posibilidad en la construcción y reconstrucción de nuevos y mejores oportunidades, y escenarios de praxis.

\section{Práctica docente: acerca de su concepto}

Al pensar en el ejercicio docente como una tarea basada netamente en la puesta en práctica de un conjunto de acciones técnicas de enseñanza, dentro de un salón de clase, que ponen en evidencia el aprendizaje de un grupo de estudiantes; esta idea expone una visión atenuada de lo que verdaderamente significa y la riqueza conceptual que esconde tras de sí. Para fortuna del ámbito de la innovación educativa, aquel supuesto que enlaza la práctica docente con la manifestación de un conjunto de técnicas de enseñanza empieza a ser revalorado, y desde allí se genera un nuevo horizonte de reflexión e interpretación que apunta a un concepto más amplio en términos de los autores; así mismo, sobre las dinámicas que hacen parte de este proceso.

Hoy, es posible avanzar sobre una reconceptualización de la práctica docente que asume al maestro y al estudiante "en su papel de sujetos que intervienen e interactúan en el proceso educativo, y no solo como insumos o productos del mismo" (Fierro et al., 1999, p. 21); de ahí, se comienza entonces a pensar en la labor educativa como una dinámica, donde cada ser humano que participa se encuentra en condiciones de aportar y a la vez
Se comenzará por abordar su definición según los aportes expuestos por autores como Fierro, Fortoul y Rosas (1999). 


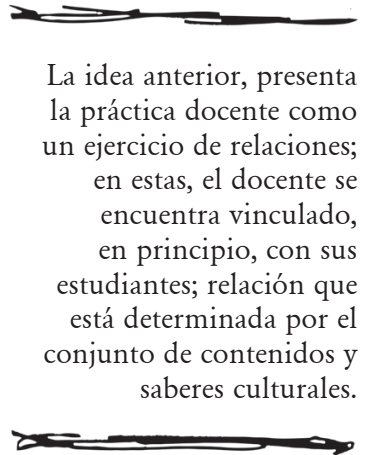

La idea anterior, presenta la práctica docente como en estas, el docente se encuentra vinculado, en principio, con sus estudiantes; relación que está determinada por el saberes culturales. de aprender. Entender la práctica docente es transitar sobre un concepto de notoria complejidad que habla de una praxis social, en la que intervienen un sinnúmero de formas de ver y comprender la realidad, mediante el acercamiento a las diferentes percepciones de los sujetos (Ruiz, Ortiz \& Soler, 2013), interpretaciones que, a su vez, son el punto de partida para descubrir nuevas formas de interpretación y reconstrucción.

La idea anterior presenta la práctica docente como un ejercicio de relaciones; en estas, el docente se encuentra vinculado, en principio, con sus estudiantes; relación que está determinada por el conjunto de contenidos y saberes culturales. En segundo lugar, es posible observar cómo el docente se encuentra cercano a la escuela, escenario donde tiene lugar el desempeño de sus funciones y que le brinda diversas oportunidades de mejora, "esto supone el ejercicio de los saberes acumulados en beneficio de la constitución como humano pero también la certeza de que su propia naturaleza encierra el movimiento hacia su transformación" (Zambrano, 2016, p. 51). Avanzando un poco más, se va descubriendo cómo el trabajo del maestro también se encuentra enlazado con valores y creencias que surgen en el contexto institucional, social y personal, toda vez que

[...] "la educación como un proceso intencional de formación de personas lleva siempre implícita una orientación hacia el logro de determinados propósitos, a través de los cuales se pretende apuntar a la formación de un determinado tipo de hombre y construir un determinado modelo de sociedad" (Fierro et al., 1999, p. 23).

Así, empieza a observarse en la práctica docente, un ejercicio que traspasa el salón de clases y transita por una serie de conexiones; en las que se encuentran estudiantes, colegas, directivos institucionales, padres de familia, saberes, comunidad educativa; $\mathrm{y}$, a nivel general, el complejo tejido de aspectos de la vida humana que construyen una sociedad.

\section{Práctica docente: sus dimensiones}

Analizar la práctica docente es una labor que exige abordar diversas aristas que invitan a salir del aula de clase y provocar un diálogo continuo, considerando aquello que está en el exterior para conectarlo con lo que se genera en el interior de un establecimiento educativo. Retomando a Carbonell (2015), se persigue, en síntesis,

[...] el impulso del diálogo entre escuela y entorno para tratar de tener puentes continuos entre el conocimiento que se produce dentro y fuera de la institución escolar, con el fin de lograr al tiempo la transferencia y el uso de la cultura escolar a la vida cotidiana y la incorporación de la experiencia vivencial y extraescolar de la escuela formal” (pp. 31-32).

En este sentido, la práctica docente entraña una relación pedagógica; aquella que promueven seis ámbitos que la describen, la construyen y, a la vez, la enriquecen a nivel conceptual. 
Se parte entonces de una dimensión personal, idea que recalca cómo la práctica docente es, ante todo, una experiencia que habla de la complejidad humana y que pone de manifiesto al docente como un profesional de la educación que, paralelamente, es sujeto de diversas vivencias fuera de su quehacer laboral. En seguida, se toma la dimensión institucional; para ir tras su lectura, se recuerda que "la institución escolar representa para el maestro, el espacio privilegiado de socialización profesional. A través de ella entra en contacto con los saberes del oficio, las tradiciones, las costumbres y las reglas tácitas propias de la cultura magisterial" (Fierro et al., 1999, p. 30). Entonces, es en la institución donde el ejercicio individual adquiere un rasgo colectivo al entrar en contacto con otros colegas, su carácter y visión de la profesión.

Además,conladimensióninterpersonal, se asume al docente como un agente que trabaja permanentemente según la interacción con el otro, en medio de un espacio colectivo que "lo pone continuamente en la necesidad de ponerse de acuerdo con otros, de tomar decisiones conjuntas, de participar en proyectos [...]" (Fierro et al., 1999, p. 32). Para avanzar en esta relación de las dimensiones de la práctica docente, se encuentra la dimensión social, cuyo contenido de análisis se fundamenta en la reflexión que hace cada maestro sobre su quehacer, teniendo en cuenta un momento histórico y un entorno particular; esta dimensión "intenta recuperar un conjunto de relaciones que se refieren a la forma en que cada docente percibe y expresa su tarea como agente educativo cuyos destinatarios son diversos sectores sociales" (Fierro et al., 1999, p. 33).

Se integra la dimensión didáctica, que describe al docente como aquel que orienta y guía el acercamiento "[...] de sus alumnos con el saber cultural a través de procesos de enseñanza. Aquí la tarea básica del maestro consiste en facilitarles el acceso al conocimiento, para que se apropien de él y lo recreen" (Fierro et al., 1999, p. 34). Finalmente, la dimensión valoral, que enfoca la práctica de cada docente como un ejercicio que da cuenta del conjunto de valores que la acompañan; el docente, "de manera intencional o inconsciente, está comunicando continuamente su forma de ver y entender el mundo, de valorar y entender las relaciones humanas, de apreciar el conocimiento y de conducir las situaciones de enseñanza" (Fierro et al., 1999, p. 35).

Así, la conexión existente entre cada una de las dimensiones que se mencionaron arriba, desencadena una relación pedagógica; vista como aquella interacción que pone de manifiesto la forma como el docente le hace frente al ejercicio de sus funciones y que determinará el provecho en cada proceso de aprendizaje de sus estudiantes. Por esto, el estudio de estas dimensiones, sus análisis y contextualización, deja que cada docente experimente en la cotidianidad de la institución escolar, esta vital relación; pues, pretende una construcción y valoración de la propia práctica, con el ánimo de renovarla y transformarla. Precisamente, cada docente consciente de su compromiso social en su rol de orientador, está llamado a convertirse en líder pedagógico.
Se parte entonces de una dimensión personal, idea que recalca cómo la práctica docente es, ante todo, una experiencia que habla de la complejidad humana y que pone de manifiesto al docente como un profesional de la educación que, paralelamente, es sujeto de diversas vivencias fuera de su quehacer laboral. 
Una de las realidades evidentes en algunas prácticas docentes, hace referencia a un "activismo sin sentido, motivado únicamente por el afán de obtener resultados (aprobados), cumplir nuestra función (pasar contenidos) o mantener ocupados a los alumnos para que no causen molestias (disciplina)"

\section{El docente: misión y liderazgo pedagógico}

Una de las realidades evidentes en algunas prácticas docentes, hace referencia a un "activismo sin sentido, motivado únicamente por el afán de obtener resultados (aprobados), cumplir nuestra función (pasar contenidos) o mantener ocupados a los alumnos para que no causen molestias (disciplina)" (Bustamante, 2006, Compromiso social y formación docente, párr. 2); realidad totalmente antagónica a lo que se desea de esta praxis, toda vez que limita la posibilidad de reflexionar, en forma crítica, sobre lo que se está haciendo, describirlo y reconstruirlo. Aquí, es importante tener claro que, no se trata de actuar porque las funciones docentes así lo exigen; a cambio, se quiere observar el desempeño, de convertirlo en una realidad susceptible de estudio y de transformación. La formación y el aprendizaje docente no terminan con la obtención de un título universitario, comienza verdaderamente cuando el profesional se enfrenta a los retos que implican adelantar procesos de enseñanza y aprendizaje con estudiantes que ya traen consigo una serie de ideas, nociones y emociones acerca del mundo.

Este aprendizaje toma aún mayor fuerza, cuando el docente asume plena reflexión sobre su forma de trabajo, y reconoce que no siempre sus estrategias serán las más efectivas; y que, por esta razón, está llamado a pensar una y otra vez en la manera de abrir nuevos horizontes. Al respecto, es necesario no perder de vista que el desarrollo docente "va más allá de su labor como instructor y tiene que ver no sólo con su rol en el entrenamiento social, sino con su compromiso con las personas de sus alumnos, en una interacción cercana y cordial." (Bustamante, 2006, Conclusión, párr. 2).

Entonces, todo docente está llamado a asumir un papel de líder pedagógico en medio de la necesidad de cambio que enfrenta durante su quehacer profesional, "Cuando el maestro, docente, educador, enseña, muestra y se muestra; se expone, se hace testimonio vivo de lo que quiere mostrar; a su vez permite al otro ser lo que es" (Gómez, Bustamante \& Castiblanco, 2015, p. 220). En ese orden de ideas, es igualmente válido saber que se encuentra ante la responsabilidad de provocar un proceso continuo de mejoramiento, teniendo en cuenta que la motivación y la toma de decisiones son de capital importancia. Esta tarea de liderazgo "consiste en crear a partir de lo que conocen los alumnos, a partir de sus conocimientos previos, estrategias que construyan sus aprendizajes, y al cabo del tiempo, lo vuelvan a "desaprenderaprender”" (Encarnación, 2012). Esto significa hacer una relación de las potencialidades del estudiante junto con sus oportunidades de mejoramiento a partir del planteamiento de una serie de objetivos.

En consecuencia, comprender el proceso de aprendizaje de sus estudiantes y reflexionar sobre su propia práctica docente, hace posible que los componentes del contexto escolar, las vivencias y experiencias, se conviertan en el mejor pretexto para nuevos y permanentes análisis, ya que las 
necesarias transformaciones en la práctica docente provienen de una presencia y participación decidida de cada docente. Se espera entonces que todo profesional de la educación que se proyecte como líder pedagógico, entiende que este

[...] no nace, se hace; el liderazgo pedagógico es algo susceptible de ser aprendido, una condición que puede ser alcanzada por aquellos docentes que sienten la necesidad de hacer bien las cosas y tienen la disposición de consagrarse al trabajo pedagógico creador, como prueba de fidelidad a una línea de acción, una profesión, una obra o una causa de marcada significación social: la educación.

\section{(Re)significación de la} práctica docente desde las
experiencias Rondonistas

La experiencia tiene en cuenta una metodología basada en la investigación cualitativa, la cual pretende "realizar un estudio sobre el quehacer cotidiano de las personas o de grupos pequeños (centrando el foco de análisis) en lo que la gente dice, piensa, siente o hace, sus patrones culturales; el proceso y significado de sus relaciones y con el medio" (Lerma, 1999, p. 40) y, apuntado hacia una línea de investigaciónacción, definida por Carr y Kemmis 1988), como "una forma de indagación autorreflexiva que emprenden los participantes en situaciones sociales en orden a mejorar la racionalidad y la justicia de sus propias prácticas, su entendimiento de las mismas y las situaciones dentro de las cuales ellas tiene lugar” (p. 174).
La Institución Educativa Juan José Rondón del municipio de Soatá, ha sido el epicentro de variadas exploraciones, observaciones y análisis sobre las prácticas docentes, adelantadas en las áreas de Matemáticas y Ciencias Naturales y Educación Ambiental para el caso de grado octavo de Educación Básica Secundaria. Algunos cuestionamientos críticos, fueron tomando fuerza en cada una de las fases de la investigación, dado que siempre se ha mantenido la atención en interpretar la realidad de este proceso a partir de la puesta en marcha de varios instrumentos, cuya pretensión radica en: explicar estas prácticas a través de un interrogante que indaga por los aspectos que propician la (re)significación de la práctica docente Rondonista, en las áreas de Matemáticas y Ciencias Naturales y Educación Ambiental para transformar las situaciones de aprendizaje en el aula con los estudiantes de grado 8-02.

Al hacer un repaso general por cada una de las fases de investigación, es preciso mencionar que, en un primer escalón, la mirada se ha centrado en explicar el estado actual de la práctica docente en las áreas mencionadas. Grabaciones de clases, análisis del discurso manejado por los docentes y encuestas semiestructuradas aparecen como protagonistas en esta ocasión. Para la segunda fase, y partiendo del planteamiento de autores como Jiménez \& Pineda (2003), Paulo Freire (1970), Gonzaga (2002), y en el caso de las Ciencias Naturales y Educación Ambiental Fierro, Fortoul, \& Rosas. (1999), De Bono (1986), y Devanand (2002); se ha adelantado un conjunto de estrategias cuya finalidad es empatar la
La Institución Educativa Juan José Rondón del municipio de Soatá, ha sido el epicentro de variadas exploraciones, observaciones y análisis sobre las prácticas docentes. 
(Re)significar la práctica docente es un concepto que convoca a cada profesional de la educación a construir reflexivamente otras maneras de afrontar esta práctica utilización de las TIC y la adecuación de ambientes de aprendizaje para transformar la práctica docente, praxis que desde el abordaje de la fase uno abrió horizontes de cualificación profesional y oportunidades de mejoramiento; en este momento, el cuaderno de notas y nuevamente las encuestas semiestructuradas apoyaron este proceso.

Durante la tercera fase, el objetivo ha sido realizar un seguimiento a la propuesta didáctica aplicada, mediante la utilidad que brinda instrumentos como las guías de observación y los registros en cuadernos de notas para el antes, durante y después de la aplicación de la misma. Para la cuarta fase, se trabaja en la interpretación y el análisis de los resultados obtenidos en cada una de las fases, tomando como punto de partida el espiral de Carr y Kemmis (1988). Dentro de ellas y haciendo una síntesis, se llega a inferir que:

- Las acciones que se adelantan para alcanzar más y mejores integraciones sociales en el aula, requieren de la valoración que se realice sobre las vivencias cotidianas de cada estudiante; de esta manera, determinar potencialidades y oportunidades de mejoramiento en los procesos de aprendizaje.

- Es preciso realizar una autoevaluación y reflexión permanente de la práctica docente, puesto que, según Jiménez et al. (2011), son la base para la resignificación, que busca generar cambios importantes en la manera de abordar procesos de enseñanza y aprendizaje.

\section{A manera de conclusión}

(Re)significar la práctica docente es un concepto que convoca a cada profesional de la educación a construir reflexivamente otras maneras de afrontar esta práctica; se parte entonces del amplio mundo de posibilidades en el contexto institucional, sugieren una transformación. (Re)significar no lleva solamente a identificar falencias. Se indica aquí que es preciso involucrarse en un tejido de acciones que partan de la identificación de una realidad, para ir más allá, para perseguir el plano de la innovación. Pineda (2003) define la resignificación de la realidad como aquel proceso mediante el cual

[...] los seres humanos perciben la realidad en la que se encuentra inmerso y, como esta percepción va cambiando de acuerdo con la aparición de innovaciones relacionadas con creaciones o recreaciones, en todos los campos del quehacer humano y como esos cambios influyen directa o indirectamente en la concepción que sobre la realidad social se tiene" (p. 113).

Recrear la práctica se convierte, entonces, en el desafío que a diario cada docente debe superar, dejando de lado imaginarios que lo ubiquen en una zona de comodidad, donde al parecer todo está dicho, todo está hecho. Si se habla de educación, siempre estaremos pendientes de un proceso que se da paralelo a los cambios vertiginosos que enfrenta la sociedad, por cuanto esta (la educación) también se convierte en un proceso abarrotado de dinámicas, que son -en todo momentoun motivo de estudio para el docente, de acuerdo con los sucesos del aula, de la 
institución y del entorno que le rodea. La realidad educativa es un complejo entramado que merece ser leído, pues no es estático y a cada momento exige interacciones oportunas que dejen al descubierto las intenciones y sentidos de nuevas construcciones en torno de la práctica docente. Se señala aquí la mirada reflexiva que insiste una y mil veces en la transformación de las prácticas docentes.

Hablar del docente como un sujeto llamado a construir no solo desde el discurso, sino a partir de la acción, la cultura y la sociedad. Esto significa incitarlo a aprovechar el conjunto de recursos y potencialidades que lo acompañan tanto a él como a los estudiantes. Así es posible afirmar que:

El educador es entonces un ser privilegiado en la construcción no sólo de la cultura, sino, como consecuencia de ella, de la sociedad, de la manera cómo sus alumnos ven al mundo, de las distintas perspectivas con que interpretan a este mundo, a la sociedad y a su existencia social e individual que otorgan un orden a su convivencia naturalmente gregaria. (Bustamante, 2006, párr. 1).

Por ende, la complejidad que enmarca el proceso de enseñanza-aprendizaje no solo se evidencia a través de los contenidos y las metodologías que se abordan en las clases, esta composición viene enmarcada por lo que va más allá y que hace referencia a la totalidad de factores que en ella circulan. Así, es necesario adentrarse en una idea sobre educación, teniendo en cuenta la multiplicidad de conceptos que se ofrecen para su comprensión. Aquí, se hace una aproximación a través de Sarramona (2000), quien expone que, para su estudio, es importante acudir a

[...] algunos principios [...]. La educación es: - Un proceso de humanización para los individuos. Supone una acción dinámica del sujeto educando con otros sujetos y con su entorno. - Se lleva a cabo de acuerdo con una escala de valores. - Proporciona las bases de la integración social de los individuos. - Constituye una dimensión básica de la cultura y garantiza la supervivencia de ésta. - Se trata de un proceso permanentemente inacabado. (p. 14).

De esta manera, la educación es un proceso eminentemente humano; a través de este

[...] aprendemos una manera de vivir, de ver al mundo, aprehendemos una cultura, una cosmogonía. Pero no es sólo eso: también es el aprendizaje de una serie de signos y significaciones, un aprendizaje de relaciones simbólicas, de un lenguaje particular, de un discurso específico que nos otorga una perspectiva para entender lo que sabemos y lo que somos capaces de hacer (Bustamante, 2006, La educación: una obra eminentemente humana, párr. 2).

Ser educador y con ello convertirse en partícipe y artífice de ambientes educativos atractivos para el estudiante, exige pensar en el objetivo de crear un escenario que le ofrezca a todos (estudiantes y docentes) alternativas nuevas al acercarse a la construcción de aprendizajes diversos y al explorar
La realidad educativa es un complejo entramado que merece ser leído, pues no es estático y a cada momento exige interacciones oportunas que dejen al descubierto las intenciones y sentidos de nuevas construcciones en torno de la práctica docente. 
e interpretar el mundo. Observar atentamente el contexto, es quizás el primer paso para avanzar sobre este objetivo, pues desde este ejercicio las personas, los lugares, los discursos y las acciones irán involucrándose de tal manera que, al final, traerán como consecuencia una lectura de lo que se está haciendo, se está logrando y de lo que se está perdiendo de vista. En seguida, sistematizar esa información dará una comprensión mucho más cercana de este trabajo, comprensión que abrirá el camino para proponer, poner en marcha, nuevamente mirar y volver a empezar con el ciclo.
Ser docente es, en suma, una labor que de ninguna manera puede abordarse como algo estático. Sería totalmente desacertado pensar en esta práctica como una acción cuyas puertas están cerradas al mejoramiento. Pensar, en el aprendizaje continuo del docente, sugiere nunca olvidar que es imperiosa la necesidad de determinar espacios. Donde el profesor -en formación o en servicio- pueda hacer conciencia de sí mismo, de su labor y del mundo y pueda confirmar su compromiso con sus alumnos y su proceso de aprendizaje, un compromiso responsable con lo que sus existencias puedan llegar a ser. (Bustamante, 2006, Compromiso social y formación docente, párr. 3).

\section{Referencias}

Bustamante, Á. (2006). Educación, compromiso social y formación docente. Revista Iberoamericana de Educación, (37). Recuperado de http://www.rieoei.org/ opinion $16 . h$ tm

CARR, W., \& Kemmis, S. (1988). Teoria crítica de la enseñanza: la investigación acción en la formación del profesorado. Barcelona: Ediciones Martínez Roca S.A.

Carbonell, J. (2015). Pedagogias del siglo XXI. Alternativas para la innovación Educativa. Barcelona: Editorial octaedro.

De Bono, E., (2000). El pensamiento lateral. Buenos aires: Editorial Paidós

Devanand, S. (2002). Junto al Maestro. INC. New York: 2022.Fierro, C.,

Fortoul, B., \& Rosas, L. (1999). Transformando la Práctica Docente. Una propuesta basada en la investigación-acción. México: Editorial Paidós.

Gómez, L., Bustamante, A., \& Castiblanco, I. (2015). Estilos pedagógicos de docentes de la Facultad de Educación de la Uptc en los proyectos pedagógicos e investigativos I y II. Praxis \& Saber, 6(12), 209-240. doi:https:// doi.org/10.19053/22160159.3770 
GonZAGA, W. (2002). La observación un medio para mejorar la práctica docente.

Revista Educare, Universidad de Costa Rica. 115-126.

Encarnación, M. Liderazgo pedagógico. (2012). Guatemala. Recuperado de https://issuu.com/marlen374/docs/issuu_liderazgo_pedagogico

Jiménez Espinosa, A., Díaz Moreno, M., \& Leguizamón Romero, J. (2011). Propuesta de modelo pedagógico para formar licenciados en matemáticas. Praxis \& Saber, 2 (3), 61-86. Recuperado de http://revistas.uptc.edu.co/index. $\mathrm{php} /$ praxis_saber/article/view/1110

Lerma, H. (2009). Metodologia de la Investigación. Propuesta, anteproyecto y proyecto. Bogotá: Ecoe Ediciones.

PinedA, R. (2003). La Resignificación de la realidad a la luz de la innovación tecnológica. Revista ABRA, 2 2(31), 111-118. Recuperado de http://www. revistas.una.ac.cr/index.php/abra/article/view/4267

Rodríguez, E, Moseueda, K. (2015). Aportes de la pedagogía de Paulo Freire, en la enseñanza de la matemática: Hacia una pedagógia liberadora de la matemática. Revista de Educación y Desarrollo Social. 9 (1).

Ruiz M., Ortiz, C., \& Soler, J. (2013). Análisis crítico de la práctica pedagógica de docentes en formación. Praxis \& Saber, 4(8), 157 - 171. doi:https://doi. org/10.19053/22160159.2656

SARRAMONA, J. (2000). Teoría de la educación. Reflexión y normativa pedagógica. Barcelona: Ariel Educación.

Zambrano Leal, A. (2016). Pedagogía y didáctica: esbozo de las diferencias, tensiones y relaciones de dos campos. Praxis \& Saber, 7(13), 45-61. doi:https:// doi.org/10.19053/22160159.4159 